\title{
A Fuzzy Set/Rule Distance for Evolving Fuzzy Anomaly Detectors
}

\author{
Jonatan Gómez and Elizabeth León
}

\begin{abstract}
This paper develops a notion of quasi distance between fuzzy sets (rule detectors) that preserves a notion of fuzzy set (rule) dominance. Such quasi distance notion is used by an evolutionary algorithm during its deterministic crowding mechanism in order to preserve diversity of the evolved fuzzy rule detectors. Moreover, the evolutionary process uses a variable length encoding that allows to tune up the fuzzy set associated to each attribute in the atomic condition of a fuzzy rule by evolving the fuzzy set parameters. Experiments with real data sets are performed and some results are reported.
\end{abstract}

\section{INTRODUCTION}

Classification is a supervised learning technique that takes labeled data samples (samples with a label indicating the class it belongs to) and generates a model (classifier) that classifies new unlabeled data samples in different predefined groups or classes [1]. Classification has been extensively studied in machine learning and data mining [1], [2], [3]. According to the number of predefined classes, the family of classification problems is divided into two types: two-class problems, there are two predefined classes in the problem; and multi-class problem, there are more than two classes in the problem. An special type of two-class problem is the anomaly classification problem (sometimes called anomaly detection).

In the anomaly classification problem, samples from just one class (usually the negative class) are given or known. Clearly, anomaly classification techniques can be applied to classifications problems where conventional two-class classifications cannot be applied. Since the anomaly classification uses only samples from the negative class for building a classifier system, its performance is in general lower than the performance reached by a conventional two-class classification technique. Therefore, an anomaly classification technique should be used when positive samples cannot be collected, as is the case of the human immune system, or when the positive space changes on time, as is the case of the computer system abnormality space (new viruses are developed daily). The most successful example of an anomaly classification system is the human immune system. The immune system does not have samples of the possible antigens it will be in contact with. The immune system produces antigen detectors just using its own cells [4], [5], [6].

There are basically three different approaches to the anomaly classification problem:

1) Positive Characterization: Negative samples (normal samples) are used for building a model of the positive

Gómez and León are with the Department of Computer and Systems Engineering at Universidad Nacional de Colombia. e-mails: \{jgomezpe,eleonguz $\} @$ unal.edu.co space (abnormal space); for example, by generating a set of rules (detectors) that can recognize positive samples (abnormal patterns) [7], [8], [9].

2) Negative characterization: Negative samples are used for building a model of the negative space; for example, a set of rules that defines the negative space [10], [11], [12].

3) Generation of artificial anomalies: Negative samples are used for generating artificial anomalies (positive samples) and a two-class classification technique is used for generating the classifier from the negative samples and the artificial anomalies [13], [14].

In particular, Dasgupta and Gonzalez proposed a positive characterization technique for evolving detectors using a genetic algorithm with a niching technique [7]. They used a real vector representation instead of binary strings. Elements of the normal/abnormal space are represented by $n$-dimensional real vectors. Positive detectors define hyper-rectangles in $\mathbb{R}^{n}$ and have high level representation as rules.

In [15], Gomez et all proposed a representation of fuzzy rule detectors (FD) to produce a fuzzy measure of deviation from normal. A genetic algorithm with deterministic crowding (DC) was used for evolving such fuzzy rule detectors. Although applied with relative success in solving some intrusion detection problems [15], this approach has some disadvantages, including the following:

1) The encoding scheme does not allow one to generate simple fuzzy rules or modify the shape of the fuzzy sets.

2) The training is time consuming since it requires a large number of generations to evolve a good set of candidate detectors.

3) The number and shape of the fuzzy sets associated with each attribute should be given in advance.

4) The search space grows exponentially in the number of attributes. If the dimension of the data set is $n$ and $k$ fuzzy sets are defined for each attribute, then the size of the chromosome is $n * k$, i.e., the size of the search space is $2^{n * k}$.

In order to remove disadvantages 1 and 2 of FD, Gomez et all [16] extended the fuzzy rule detector definition and used a twolevel Structured Genetic Algorithm (StGA) representation [17] for encoding such fuzzy rule detectors into the chromosome of an individual. They called such approach Structured Fuzzy Detection (S-FD). The high level bits correspond to the set of indexes (features used) while the low level bits represent the condition part of the fuzzy rule detector in the same way that was used in FD. The length of the high level portion of 
the chromosome is the same as the dimension of the feature space, $n$. Each bit in the high level portion of the chromosome indicates whether a feature is used in the condition or not. Although the complexity of the evolved fuzzy rule detectors and evolution time is lower using S-FD than FD, disadvantages 3 and 4 are still present in the S-FD approach.

This paper proposes an encoding technique, based on the mechanism proposed by Gomez in [18], [19], that allows to evolve simple and tuned fuzzy rule detectors. Instead of using a fixed chromosome length for evolving the condition part of the fuzzy rule detectors, a variable length chromosome is used. Such condition is a conjunction of atomic expressions, each one defined in terms of a single attribute and a set of parameters defining the fuzzy set. Also, a notion of fuzzy rule distance is introduced in order to apply deterministic crowding to the evolutionary process. This paper is organized in five sections. Section 2 presents the proposed approach; Section 3 presents experiments performed on well-known machine learning data sets; Section 4 reports some experimental results with the $10 \%$ kddcup99 intrusion detection data set; Section 5 draws some conclusions.

\section{Proposed Approach (T-FD)}

\section{A. Fuzzy Set Distance}

We defined a quasi distance instead of defining a distance for fuzzy sets. A quasi distance has the same properties of a distance but without requiring the symmetry property. Given two sets $s_{1}$ and $s_{2}$, the distance from $s_{1}$ to $s_{2}\left(d\left(s_{1}, s_{2}\right)\right)$ is defined as the proportion of elements of $s_{2}$ that are not in $s_{1}$. Let $\mu_{S}, \mu_{T}: U \rightarrow[0,1]$ be the membership functions of the fuzzy sets $S$ and $T$ both of them in the same discrete universe $U$. The distance of $S$ to $T$ is defined as follows:

$$
d(S, T)=\sum_{x \in U} \max \left\{\mu_{S}(x)-\mu_{T}(x), 0\right\}
$$

This definition can be appropriately extended to continuous universes by replacing the summation with an integration operator. For example, if $U=[0,1]$, then the distance from fuzzy set $S$ to fuzzy set $T$ is defined as follows:

$$
d(S, T)=\int_{0}^{1} \max \left\{\mu_{S}(x)-\mu_{T}(x), 0\right\} d x
$$

According to equation 2, the distance between fuzzy sets $S$ and $T$ is equivalent to the area under the membership function of $S\left(\mu_{S}\right)$ that is not under the membership function of $T$ $\left(\mu_{T}\right)$. Therefore, it is possible to prove that the distance from a fuzzy set $S$ to a fuzzy set $T$ is 0 if the $\mu_{S}(x) \leq \mu_{T}(x)$ for all $x \in U$. It is said that $T$ dominates $S$ and $S$ is dominated by $T$ when $d(S, T)=0$.

\section{B. Fuzzy Rule Distance}

In this work, a fuzzy rule (detector) is defined as follows:

$$
\text { If } x_{i_{1}} \in T_{i_{1}} \wedge \ldots \wedge x_{i_{p}} \in T_{i_{p}} \text { then } x \text { is abnormal }
$$

Here, $x=\left(x_{1}, \ldots x_{n}\right) \in \mathbb{R}^{n}$ is an element of the normal/abnormal space being evaluated, $\left\{i_{k}\right\}_{k=1, \ldots, p} \subseteq$

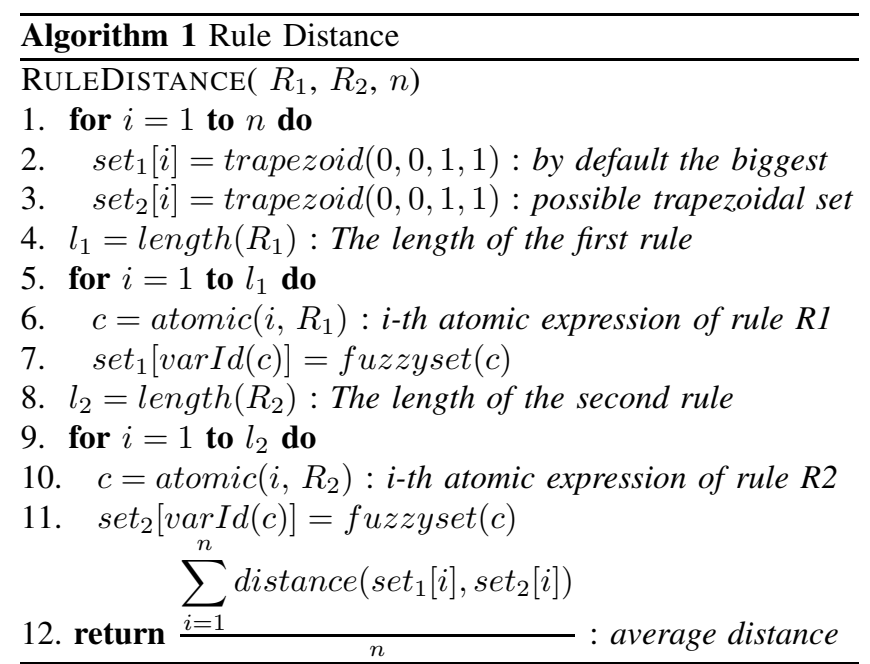

$\{1,2, . ., n\}$ is a set of indexes, $T_{i_{k}}$ is a fuzzy set, and $\wedge$ is the min-and fuzzy conjunction operator. Notice that any fuzzy rule can be extended in such a way that it includes an atomic condition (An expression having the form $x_{i} \in T_{i}$ ) for each attribute: If the condition of a fuzzy rule $R_{1}$ is defined in terms of $k$ atomic expressions $(k \leq n), a_{1}$ AND...AND $a_{k}$, none being defined in terms of the $i$ th attribute, then such condition is equivalent to the condition $a_{1}$ AND...AND $a_{m}$ AND $x_{i}$ is trapezoid $(0,0,1,1)$. This process can be repeated until all attributes are included in the condition part of the fuzzy rule. Using this property, the distance from a fuzzy rule $R_{1}$ to a fuzzy rule $R_{2}$ can be defined in different ways, see line 12 of algorithm 1 . In this paper we defined it as the average distance between the fuzzy sets of its attributes to the fuzzy sets of the attributes of rule $R_{2}$. We used the average volume instead of the real volume (volume product) since the average volume is easier to evaluate and it is affected in a lower proportion by the problem dimensionality than the product volume.

Notice that this fuzzy rule distance extends the domination definition of fuzzy sets to fuzzy rules, i.e., it is possible to prove that the distance from fuzzy rule $R_{1}$ to fuzzy rule $R_{2}$ is 0 if the degree of abnormally predicted by $R_{1}$, for any sample $x$, is higher than the degree of abnormally predicted by $R_{2}$. In other words, anything that $R_{2}$ predicts as abnormal is predicted by $R_{1}$ as abnormal too. It is said that $R_{1}$ dominates $R_{2}$ and $R_{2}$ is dominated by $R_{1}$ when $d\left(R_{1}, R_{2}\right)=0$.

\section{Encoding}

Notice that, the fuzzy rule definition proposed here, can be seen as a particular case of the Extended Conjunctive Normal Form (XCNF-1) introduced by Gomez in [18], [19] (by restricting each atomic expression to be positive), and it can be seen as a generalization of the S-FD one [16]. Therefore, in order to "virtually" reduce the search space size and allow the evolutionary algorithm to tune the fuzzy sets associated with each attribute, we use the variable-length chromosome with fuzzy set tuning encoding mechanism of fuzzy rules proposed in [18], [19]. Therefore, a fuzzy rule detector can be encoded as shown in figure 1. 


\begin{tabular}{|c|c|c|c|c|c|c|c|c|c|}
\hline \multicolumn{3}{|c|}{ Gene $_{1}$} & $\ldots$ & \multicolumn{3}{|c|}{ Gene $_{p-1}$} & \multicolumn{3}{|c|}{ Gene $_{p}$} \\
\hline \multicolumn{3}{|c|}{ Atom $_{1}$} & $\ldots$ & \multicolumn{3}{|c|}{ Atom $_{p-1}$} & \multicolumn{3}{|c|}{ Atom $_{p}$} \\
\hline$x_{i_{1}}$ & $\bar{\epsilon}$ & $\overline{T_{i_{1}}}$ & $\ldots$ & $\overline{x_{i_{p-1}}}$ & $\epsilon$ & $\overline{T_{i_{p-1}}}$ & $\overline{x_{i_{p-1}}}$ & $\epsilon$ & $\overline{T_{i_{p}}}$ \\
\hline
\end{tabular}

Fig. 1. Fuzzy Rule Detector Encoding

A set of parameters defining a fuzzy set can be encoded into each gene allowing the Evolutionary Algorithm to tune such fuzzy set. In this work, the fuzzy set tuning is restricted to trapezoidal fuzzy sets ${ }^{1}$. It is done by dividing the attribute space into $m$ regions of the same length ( $m$ is a parameter given by the user). This division generates $m+1$ control points, see Figure 2.a. Given two control points, $x$ and $y$ $(x \leq y)$, it is possible to define the trapezoidal fuzzy set: $\left(\max \left\{0, \frac{x-1}{m}\right\}, \frac{x}{m}, \frac{y}{m}, \min \left\{1, \frac{y+1}{m}\right\}\right)$, see Figure $2 . b^{2}$. Therefore, $2\left\lceil\log _{2}(m+1)\right\rceil$ bits are used for representing the two control points of the trapezoidal fuzzy set.

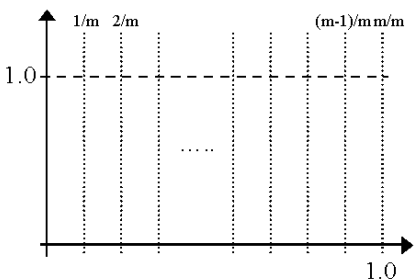

(a)

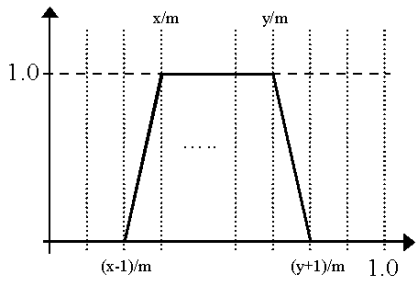

(b) Two control points trapezoid
Fig. 2. Tuning of Trapezoidal membership functions. (a) Division of the fuzzy

The genetic operators used to evolve such fuzzy rule detectors are the same as proposed in [18], [19]:

1) Variable Length Simple Point Crossover (VLSPX)

2) Single Bit Mutation (SBM)

3) Gene Addition (ADD)

4) Extended Gene Deletion (XDEL)

\section{Fitness Function}

The fitness function on T-FD is the same fitness function used by Gomez for evolving fuzzy rules in [18], [19]. Since no samples of the positive class are given, the fitness function reduces to equation 3 .

$f i t(R)=w * \frac{N N(R)}{N N(R)+N P(R)}+(1-w) *\left(1-\frac{F R L(R)}{M}\right)$

Here, $N N(N P)$ is the number of negative samples considered negative (positive), $w$ is the weight associated with the fuzzy accuracy, $F R L$ is the length of the fuzzy rule, and $M$ is the maximum number of atomic expressions defining a fuzzy rule.

\footnotetext{
${ }^{1}$ We used symmetric trapezoidal fuzzy sets as illustrative example of the encoding mechanism. The proposed method is not restricted to symmetric trapezoidal fuzzy sets.

${ }^{2}$ It will define a triangular fuzzy set instead of a trapezoidal if $x=y$.
}

\section{E. Additional Considerations}

Although DC requires a notion of distance between individuals [20], we extend the DC in order to use a quasidistance instead of a distance. Two additional issues must be tackled before being able to use T-FD: maintaining diversity, and allowing a fuzzy rule detector to cover additional non/self area with the same condition length.

1) Maintaining Diversity: Since the size of the search space is "virtually" reduced by introducing a variable length representation, the following condition is introduced in the deterministic crowding in order to reduce the crossover interaction problem:

IF (fitness $($ child $)>$ fitness (parent) AND

(child dominates parent OR child is dominated by parent))

THEN replace(parent, child)

Such a condition allows the replacement of a fuzzy rule detector that dominates or is dominated by an offspring with higher fitness.

2) Increasing a Fuzzy Rule Detector Volume: Suppose that in a given generation of T-FD, a genetic operator, like mutation, produces a offspring fuzzy rule $R_{2}$ from a fuzzy rule $R_{1}$ such that $R_{2}$ dominates $R_{1}$, the length of $R_{1}$ is equal to the length of $R_{2}$ and the fuzzy accuracy of both of them is the same. Then, the fitness of $R_{1}$ is equal to the fitness of $R_{2}$ and $R_{1}$ will not be replaced by $R_{2}$ regardless that $R_{2}$ covers more non/self space than $R_{1}$. In order to allow the replacement of individuals with the same fitness that are dominated by other, the following replacement condition is introduced in the DC:

IF $($ fitness $($ child $)=$ fitness $($ parent $)$ AND

child dominates parent THEN replace( parent, child)

\section{F. Extracting the Final Set of Fuzzy Rule Detectors}

Fuzzy rule detectors evolved by the EA (FD, S-FD or TFD) are sorted by fitness from highest to lowest. Detectors with a fitness lower than the population average are removed. The average fitness is a good indicative of the "performance" of the detectors. Then, detectors that are dominated by other detectors with a higher fitness are removed because they are not covering additional non-self space and can cover self space. Finally, detectors that are dominated, regardless their fitness, are removed.

\section{G. Normal/Abnormal Discrimination}

After the fuzzy rule detectors are generated, they are used for determining the abnormality level of an element. An element $x$ is classified as abnormal with a degree of abnormality equal to the highest truth value of the fuzzy rule detectors evaluated on such an element. A value close to zero means that $x$ is normal and a value close to one indicates that it is abnormal.

\section{Experimentation with Machine LeARning Data}

Three benchmark data sets (public-ally available), were used as a testbed. See table I. 
TABLE I

Machine LeARning Testbed

\begin{tabular}{|c|c|c|c|}
\hline DATA SET & \multirow{2}{*}{ DIM } & \multicolumn{2}{|c|}{ SAMPLES } \\
\cline { 3 - 4 } & & TOTAL & PER CLASS \\
\hline \hline BREAST & 9 & 699 & $\{458,241\}$ \\
\hline PIMA & 8 & 768 & $\{500,268\}$ \\
\hline HEART & 13 & 270 & $\{150,120\}$ \\
\hline
\end{tabular}

\section{A. Experimental Settings}

The data set is divided into 10 random groups of similar size. Then, each group is used as testing set of the fuzzy rule evolved by FD, S-FD and T-FD using the normal samples of the remaining 9 groups as a training set. This validation techniques can be seen as a 10 cross validation without using the abnormal samples for training purposes, but only for testing. This procedure is repeated 5 times and the reported results are the average of such 50 runs. Each evolutionary algorithm is run for a maximum of 30 generations with a population size of 100 individuals. The accuracy weight is set to $w=0.9$ (in order to give more importance to the correct discrimination of the normal samples than to the area covered by the fuzzy rule detector), the Min-And fuzzy operator is used, single point crossover and single bit mutation are used by the FD and S-FD approaches while single bit mutation (SBM), variable length crossover (VLSPX), gene addition (ADD) and extended gene deletion (XDEL) are used by the T-FD approach. Also, we associated 5 fuzzy sets to each attribute in the FD and S-FD approaches while we associated 6 divisions to each fuzzy set parameter in T-FD (to perform a fair comparison between T-FD and S-FD). Finally, each chromosome in the T-FD approach is initialized with a random length between 1 and $n$ genes ( $n$ is the number of different attributes).

\section{B. Results and Analysis}

1) Comparison between $T-F D, S-F D$, and FD: Table II presents the average accuracy reached by T-FD, S-FD and FD, along with the average number of fuzzy rule detectors generated by each approach. A value of $a \pm b$ indicates that the average values was $a$ with standard deviation of $b$.

As expected, the performance of FD is low compared to the performance of T-FD and S-FD since it is not able to explore appropriately the search space in few generations. Thus, FD generates a high number of detectors compared to the number of detectors generated by S-FD and T-FD. Notice that T-FD performs similarly to S-FD since the dimensionality of these data sets is low (less than 14 dimensions).

2) Fuzzy Rule Complexity: Although the T-FD and S-FD performance is almost the same, T-FD generates more fuzzy rule detectors than S-FD. However, the complexity of the detectors generated by T-FD is low compared to the detectors generated by S-FD. While a fuzzy rule detector in S-FD can represent a non-connected region, a T-FD fuzzy rule detector always represents a connected region. Here, T-FD scores in interpretability. Moreover, a fuzzy rule detector generated by S-FD is equivalent to many fuzzy rule detectors generated by T-FD.
Figure 3 shows the evolution of the fuzzy rule length on TFD for the best individual and the average of the population. Clearly, T-FD produces simple fuzzy rules, as no more than 3 attributes are included in the condition part of the fuzzy rules.

The following is a fuzzy rule detector generated by T-FD for the HEART data set in a sample run:

$$
\text { IF } x_{12} \text { is set } \text { s, }_{25} \text { AND } x_{3} \text { is set } \text { s,6 }_{2} \text { THEN Heart-Disease }
$$

Here, set s,y $_{x}$ represents the trapezoidal fuzzy $\left(\max \left\{0, \frac{x-1}{m}\right\}, \frac{x}{m}, \frac{y}{m}, \min \left\{1, \frac{y+1}{m}\right\}\right)$. As can be noticed, the evolved fuzzy rules are easily interpretable by a human being.

3) Comparison with Results Reported in the Literature: Rows 1 and 2 of Table III presents the average performance reached by T-FD and S-FD along with some results reported in the literature and the fuzzy rule evolutionary approach (EFR) proposed by Gomez et all in [18], [19] (third row) ${ }^{3}$.

TABLE III

COMPARATIVE PERFORMANCE OF THE PROPOSED APPROACH

\begin{tabular}{|c|c|c|c|c|}
\hline Method & BREAST & PIMA & HEART & Statistical Test \\
\hline \hline T-FD & $\mathbf{9 2 . 8 5}$ & $\mathbf{6 5 . 4 7}$ & $\mathbf{6 2 . 4 4}$ & $\mathbf{1 0 - c r o s s - v a l i d a t i o n ~}$ \\
\hline S-FD & $\mathbf{9 2 . 7 4}$ & $\mathbf{6 6 . 7 5}$ & $\mathbf{6 1 . 1 1}$ & $\mathbf{1 0 - c r o s s - v a l i d a t i o n ~}$ \\
\hline EFR & $\mathbf{9 4 . 8 5}$ & $\mathbf{7 4 . 2 1}$ & $\mathbf{7 8 . 9 6}$ & 10-cross-validation \\
\hline QDA & 94.90 & 74.80 & 57.80 & Leave-one-out \\
\hline LDA & 96.00 & 77.20 & 60.40 & Leave-one-out \\
\hline C4.5 & 94.70 & 73.00 & 22.90 & Leave-one-out \\
\hline kNN & 96.90 & 71.90 & 65.60 & Leave-one-out \\
\hline SSV & 96.30 & 73.70 & - & 10 -cross-validation \\
\hline FSM & 96.90 & - & - & 10 -cross-validation \\
\hline WM & 87.10 & 71.30 & - & - \\
\hline GIL & 90.10 & 73.10 & - & - \\
\hline ABD & 96.00 & 75.90 & - & - \\
\hline ABA & 95.10 & 74.80 & - & random $(50-50) \%$ \\
\hline
\end{tabular}

As expected, EFR performs better than T-FD and S-FD because it has access to the abnormal information during the training process. This information allows EFR to produce a better characterization of the normal/abnormal boundary. Considering that T-FD and S-FD have only access to the normal information, results for T-FD and S-FD can be considered good; such results are similar to some results reported in the literature when using all samples (normal and abnormal samples) $)^{4}$.

\section{EXPERIMENTATION WITH INTRUSION DETECTION DATA SETS}

\section{A. The 10\% KDDCup99 Data Set}

This data set is $10 \%$ of the KDDCup99 data set [22] which is a version of the 1998 DARPA intrusion detection evaluation data set prepared and managed by MIT Lincon Labs [23]. In this data set, 42 attributes (or fields) that usually characterize network traffic behavior compose each record. Some of these attributes are categorical (8) while others are

\footnotetext{
${ }^{3}$ Results reported for QDA, LDA, C4.5, kNN, SSV and FSM taken from [21].

${ }^{4}$ Although all of these results were obtained using different portions of the data set and with different statistical validation methods (leave-one-out, or 10 -cross-validation) or without statistical validation, the values reported here are indicative of the performance of the proposed approach.
} 
TABLE II

COMPARATIVE PERFORMANCE OF T-FD AND S-FD .

\begin{tabular}{|c|c|c|c|c|c|c|}
\cline { 2 - 7 } \multicolumn{1}{c|}{} & \multicolumn{2}{c|}{ T-FD } & \multicolumn{2}{c|}{ S-FD } & \multicolumn{2}{c|}{ FD } \\
\cline { 2 - 7 } \multicolumn{1}{c|}{} & Accuracy \% & Detectors & Accuracy \% & Detectors & Accuracy \% & Detectors \\
\hline BREAST & $\mathbf{9 2 . 8 5} \pm \mathbf{3 . 6 1}$ & $\mathbf{3 1 . 7 8} \pm \mathbf{5 . 2 7}$ & $92.74 \pm 4.10$ & $18.18 \pm 6.40$ & $84.12 \pm 5.06$ & $97.96 \pm 2.69$ \\
\hline PIMA & $\mathbf{6 5 . 4 7} \pm \mathbf{4 . 6 6}$ & $\mathbf{4 2 . 3 8} \pm \mathbf{4 . 1 9}$ & $66.75 \pm 4.58$ & $18.82 \pm 7.47$ & $65.73 \pm 2.55$ & $98.28 \pm 1.86$ \\
\hline HEART & $\mathbf{6 2 . 4 4} \pm \mathbf{9 . 0 4}$ & $\mathbf{3 8 . 1 0} \pm \mathbf{4 . 6 0}$ & $61.11 \pm 7.99$ & $29.60 \pm 10.40$ & $60.22 \pm 6.66$ & $99.20 \pm 0.97$ \\
\hline
\end{tabular}

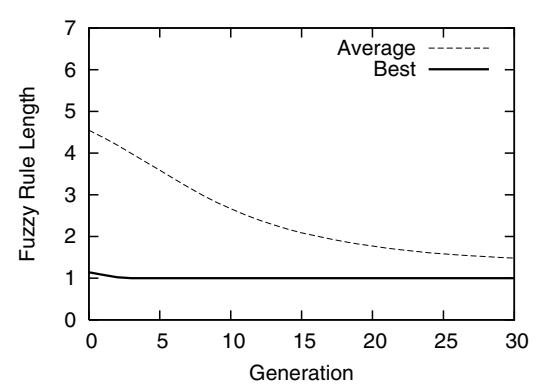

(a)

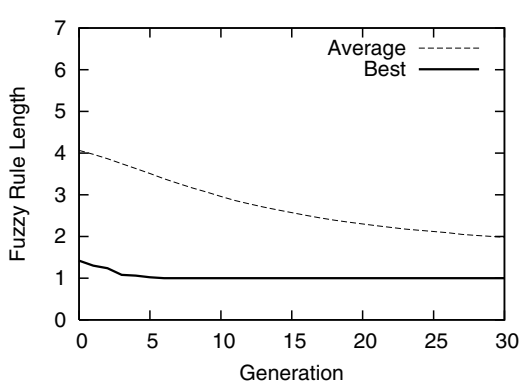

(b)

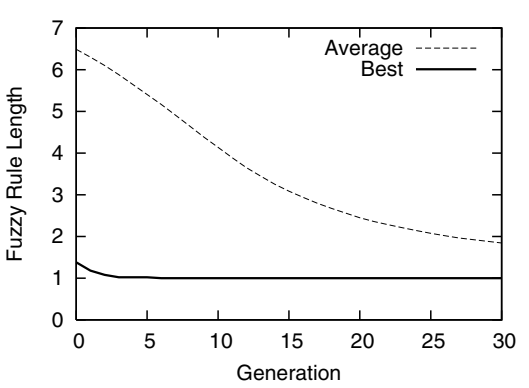

(c)

Fig. 3. Fuzzy Rule Length Evolution. (a) BREAST, (b) PIMA, (c) HEART.

discrete or numerical (34). The total number of records in the $10 \%$ data set is 492021 .

This data set contains 22 different types of attacks that can be classified in four main intrusion classes, as is shown in table IV. The proportion of samples per class is not uniform; for example from class U2R the number of samples in the training data set is 59 while from class DOS the number of samples is 391458 .

TABLE IV

Classes IN $10 \%$ OF THE KDDCuP 99

\begin{tabular}{|c|l|c|c|}
\hline CLASS & \multicolumn{1}{|c|}{ SUB-CLASSES } & SAMPLES & \% \\
\hline \hline Normal & \multicolumn{1}{|c|}{} & 95278 & $19.3 \%$ \\
\hline U2R & $\begin{array}{l}\text { buffer_overflow, loadmodule, } \\
\text { multihop, perl, rootkit }\end{array}$ & 59 & $0.01 \%$ \\
\hline R2L & $\begin{array}{l}\text { ftp_write, guess_passwd, imap, phf, } \\
\text { spy, warezclient, warezmaster }\end{array}$ & 1119 & $0.23 \%$ \\
\hline DOS & $\begin{array}{l}\text { back, land, neptune, pod, smurf, } \\
\text { teardrop }\end{array}$ & 391458 & $79.5 \%$ \\
\hline PRB & ipsweep, nmap, portsweep, satan & 4107 & $0.83 \%$ \\
\hline
\end{tabular}

This data set was prepared in such a way that only the numerical attributes were used in the training-testing process. Moreover, a numerical attribute was removed since it was the same value for each record in the data set. Therefore, the reduced version of the KDDCup 99 data set contains 33 attributes.

\section{B. Experimental settings}

A collection of 2000 normal data records is drawn from the data set and used as training data set while the remaining part of the data set is used as testing data. The score of the trained classifier is calculated as the classification accuracy over the testing set. This process is repeated 10 times and the reported results are the average of these 10 runs. The accuracy weight is set to $w=0.9$, Min-And fuzzy operator are used, single bit mutation (SBM), variable length crossover (VLSPX), gene addition (ADD) and extended gene deletion (XDEL) gene are used in the T-FD approach while single bit mutation and single point crossover are applied in the S-FD approach. Also, 6 divisions are allowed for tuning a fuzzy set while 5 fuzzy sets are associated to each attribute in the S-FD approach. The performance of T-FD and S-FD is calculated after 30, 50,80 and 100 generations of the evolutionary algorithm with a population size of 100 individuals. These experiments are conducted in order to determine the effect of number of iterations in the quality of the solution produced by the those approaches (T-FD and S-FD).

\section{Results and Analysis}

Figures 4.a-d show the ROC curves generated by T-FD and $\mathrm{S}-\mathrm{FD}$ when the maximum number of generations was set to 30, 50, 80 and 100 .

Clearly, T-FD outperforms S-FD regardless of the number of generations. Since the variable length encoding mechanism of T-FD reduces the search space size, it is able to evolve fuzzy rule detectors that cover large regions of the abnormal space in a few generations while keeping the false alarm rate low. It is not the case of S-FD, since the search space of SFD is huge. It produces fuzzy rule detectors that cover small regions of the abnormal space. Table $\mathrm{V}$ compares the detection rate reached by S-FD and T-FD when the false alarm rate is fixed to $5 \%$ along with the number of fuzzy rule detectors generated. Figure 4.e compares the ROC curves generated by T-FD after different number of generations. When the number of generations is increased, T-FD is able to increase the detection rate while keeping the false alarm rate low. 


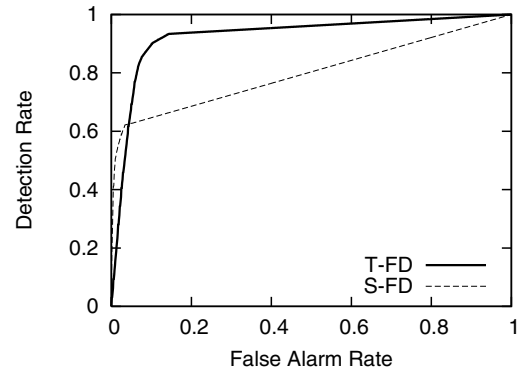

(a)

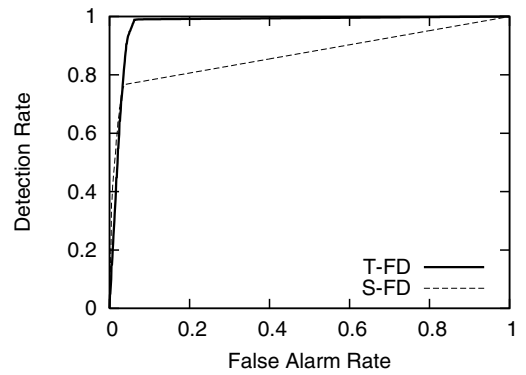

(b)

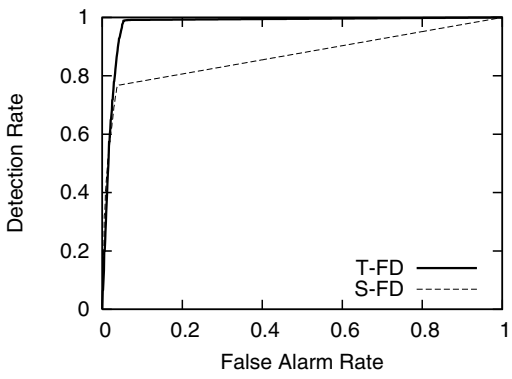

(c)

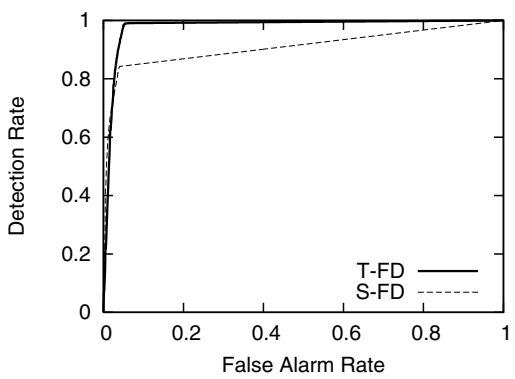

(d)

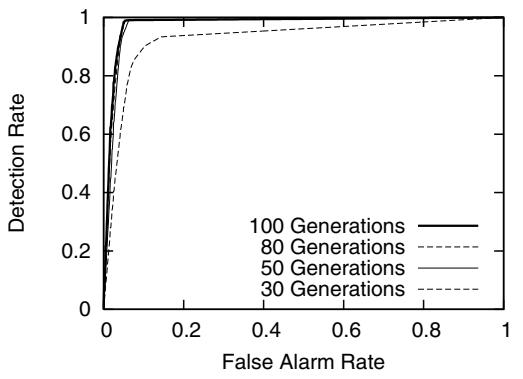

(e)

Fig. 4. ROC curves generated by T-FD and S-FD on the KDDCup 99 data set when they were allowed to evolve fuzzy rule detectors for a maximum of (a) 30, (b) 50, (c) 80 and (d) 100 generations. (e) ROC curves generated by T-FD on the KDDCup 99 data set after different number of generations.

TABLE V

Detection RATE of T-FD AND S-FD in THE KDD CuP 99 PROBLEM WHEN THE FALSE ALARM IS SET TO $5 \%$.

\begin{tabular}{|c|c|c|c|c|}
\cline { 2 - 5 } \multicolumn{1}{c|}{} & \multicolumn{2}{c|}{ T-FD } & \multicolumn{2}{c|}{ S-FD } \\
\hline Geners & DR \% & Detectors & DR \% & Detectors \\
\hline \hline $\mathbf{3 0}$ & $\mathbf{6 9 . 2 0} \pm \mathbf{2 3 . 0 1}$ & $\mathbf{4 5 . 9} \pm \mathbf{3 . 6 3}$ & $62.72 \pm 34.61$ & $76.6 \pm 12.67$ \\
\hline $\mathbf{5 0}$ & $\mathbf{9 4 . 7 5} \pm \mathbf{7 . 0 6}$ & $\mathbf{4 3 . 6} \pm \mathbf{3 . 5 7}$ & $77.02 \pm 31.33$ & $42.5 \pm 20.42$ \\
\hline $\mathbf{8 0}$ & $\mathbf{9 7 . 6 0} \pm \mathbf{1 . 8 5}$ & $\mathbf{4 0 . 4} \pm \mathbf{3 . 7 5}$ & $77.03 \pm 32.70$ & $26.5 \pm 11.21$ \\
\hline $\mathbf{1 0 0}$ & $\mathbf{9 8 . 2 8} \pm \mathbf{1 . 5 6}$ & $\mathbf{4 0 . 9} \pm \mathbf{4 . 1 2}$ & $84.41 \pm 28.28$ & $23.0 \pm 8.11$ \\
\hline
\end{tabular}

If each attack is considered independently, T-FD generates the ROC curves shown in Figure 5 after evolving fuzzy rule detectors for 100 generations.

As expected, the behavior of the ROC curve considering only $D o S$ attack is almost the same as considering all the attacks. This is due to the fact that $D o S$ includes more than $99 \%$ of the attacks.

\section{Conclusions}

This paper presented a new technique that allows to generate a set of fuzzy rules that characterize the abnormal space using as input only normal samples. This work introduces the basic idea of evolution of fuzzy rule detectors using the mechanism of variable length and fuzzy set tuning proposed in [18], [19]. These extensions are developed in order to eliminate some disadvantages of our previous approaches of fuzzy normal/abnormal discrimination techniques. Experiments showed that it performs better than the previous ones. The following are the main advantages of the fuzzy Normal/Abnormal discrimination technique introduced:

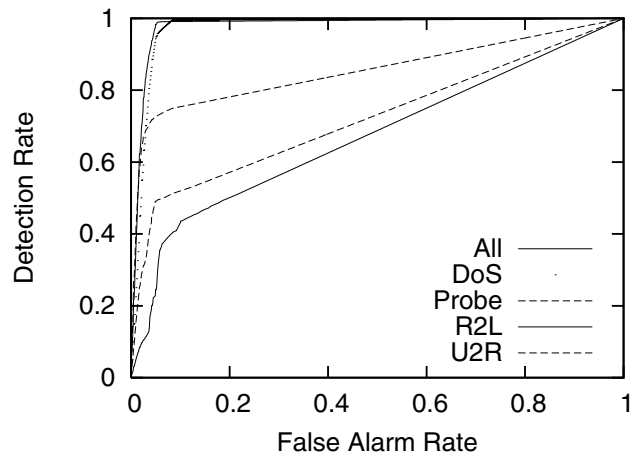

Fig. 5. ROC Curve generated by T-FD for each different attack.

- It shows an improved accuracy in the anomaly detection problem. This can be attributed to the fuzzy representation of rules which reduces the search space, allowing the evolutionary algorithm to find better solutions.

- It generates a more compact representation of the nonself space by reducing the number of detectors. This is also a consequence of the expressiveness of the fuzzy rules and the variable length encoding mechanism.

- It speeds up the evolutionary process and improves the performance of the algorithm by using variable length representation for the fuzzy rules.

Our future work will to develop cooperative behavior of the fuzzy rule detectors for covering large regions of the abnormal space (co-evolution). 


\section{REFERENCES}

[1] J. Han and M. Kamber, Data Mining: Concepts and Techniques. Morgan Kaufmann, 2000.

[2] R. S. Michalski, I. Bratko, and M. Kubat, Machine Learning and Data Mining: Methods and Applications. J. Wiley \& Sons, 1998.

[3] R. Holte, "Very simple classification rules perform well in most common used datasets," Machine Learning, no. 11, pp. 63-91, 1993.

[4] A. Tyrrell, "Computer know thy self! : a biological way to look at fault tolerance," in Proceedings of the 2nd Euromicro/leee workshop on Dependable Computing Systems, (Milan), pp. 129-135, 1999.

[5] F. Varela, A. Coutinho, B. Dupire, and N. Vaz, "Cognitive networks: immune and neural and otherwise," Theoretical Immunology: Part Two, SFI Studies in the Sciences of Complexity, pp. 359-371, 1988.

[6] J. Timmis, Artificial Immune Systems: a Novel Data Analysis Technique Inspired by the Immune Network Theory. $\mathrm{PhD}$ thesis, University of Wale, 2000

[7] D. Dasgupta and F. Gonzalez, "An immunity-based technique to characterize intrusions in computer networks," IEEE Transactions on Evolutionary Computation, vol. 6, pp. 281-291, 1999.

[8] F. Gonzalez and D. Dasgupta, "An immunogenetic technique to detect anomalies in network traffic," in Proceedings of the genetic and evolutionary compuation conference, GECCO 2002, pp. 1081-1088, Morgan Kaufman Publishers, 2002.

[9] D. Dasgupta and S. Forrest, "An anomaly detection algorithm inspired by the immune system," Artificial immune systems and their applications, pp. 262-277, 1999.

[10] W. Lee, S. Stolfo, and K. Mok, "Mining Audit Data to Build Intrusion Detection Models," in International Conference Knowledge Discovery and Data Mining (KDD'98), pp. 66-72, 1998.

[11] S. Bridges and R. Vaughn, "Fuzzy Data Mining and Genetic Algorithms Applied to Intrusion Detection," in Twenty Third National Information Security Conference, Oct. 2000.

[12] S. Hofmeyr, A. Somayaji, and S. Forrest, "Intrusion Detection Using Sequences of Systems Call," Computer Security, no. 6, pp. 151-180, 1998.

[13] W. Fan, W. Lee, M. Miller, S. Stolfo, and P. Chan, "Using artificial anomalies to detect unknown and known network intrusions," in Proceedings of the first IEEE International conference on Data Mining, 2001.

[14] F. Gonzalez and D. Dasgupta, "Neuro-immune and self-organising map approaches to anomaly detection: A comparison," in First International Conference on Artificial Immune Systems, Sept. 2002.

[15] J. Gomez, F. Gonzalez, and D. Dasgupta, "An immuno-fuzzy approach to anomaly detection," in Proceedings of the IEEE International Conference on Fuzzy Systems FUZZIEEE 2003, 2003.

[16] J. Gomez, F. Gonzalez, M. Kaniganti, and D. Dasgupta, "An evolutionary approach to generate fuzzy anomaly (attack) signatures," in Proceedings of the IEEE Information Assurance Workshop 2003 Conference, 2003.

[17] D. Dasgupta and D. R. McGregor, "Non-stationary function optimization using the structured genetic algorithm,"

[18] J. Gomez, "A framework for evolving fuzzy rule classifiers," in Proceedings of the Congress on Evolutionary Computation 2004, 2004.

[19] J. Gomez, "Evolution of fuzzy rule based classifiers," in Proceedings of the Genetic and Evolutionary Computation Conference GECCO 2004, 2004.

[20] S. W. Mahfoud, "Crowding and preselection revisited," in Proceedings Second Conference Parallel Problem Solving from Nature, 1992.

[21] D. Wlodzislaw, "Data sets used for classification: Comparison of results," in http://www.phys.uni.torun.pl/kmk/projects/datasets.html.

[22] "Kdd-cup data set." http://kdd.ics.uci.edu/databases/kddcup99/kddcup99.html.

[23] "Lincoln laboratory mit.." http://www.ll.mi.edu/. 